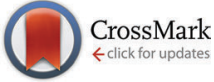

Cite this: Phys. Chem. Chem. Phys., 2017, 19, 10786

Received 9th December 2016, Accepted 15th February 2017

DOI: $10.1039 / c 6 c p 08436 b$

rsc.li/pccp

\title{
Photodissociation spectroscopy of protonated leucine enkephalin $\dagger$
}

\begin{abstract}
Andreas Herburger, Christian van der Linde and Martin K. Beyer*
Protonated leucine enkephalin (YGGFL) was studied by ultraviolet photodissociation (UVPD) from 225 to $300 \mathrm{~nm}$ utilizing an optical parametric oscillator tunable wavelength laser system (OPO). Fragments were identified by absolute mass measurement in a $9.4 \mathrm{~T}$ Fourier transform ion cyclotron resonance mass spectrometer (FT-ICR MS). Bond cleavage was preferred in the vicinity of the two aromatic residues, resulting in high ion abundances for $a_{4}, a_{1}, b_{3}, y_{2}$ and $y_{1}$ fragments. $a, b$ and $y$ ions dominated the mass spectrum, and full sequence coverage was achieved for those types. Photodissociation was most effective at the short wavelength end of the studied range, which is assigned to the onset of the $L_{a} \pi-\pi^{*}$ transition of the tyrosine chromophore, but worked well also at the $L_{b} \pi-\pi^{\star}$ chromophore absorption maxima in the $35000-39000 \mathrm{~cm}^{-1}$ region. Several side-chain and internal fragments were observed. $\mathrm{H}$ atom loss is observed only above $41000 \mathrm{~cm}^{-1}$, consistent with the requirement of a curve crossing to a repulsive ${ }^{1} \pi \sigma^{\star}$ state. It is suggested that the photochemically generated mobile $\mathrm{H}$ atom plays a role in further backbone cleavages, similar to the mechanism for electron capture dissociation. The $b_{4}$ fragment is most intense at the $L_{b}$ chromophore absorptions, undergoing additional fragmentation at higher photon energies. The high resolution of the FT-ICR MS revealed that out of all $x$ and $z$-type fragments only $x_{3}$ and $x_{4}$ were formed, with low intensity. Other previously reported $x$ - and $z$-fragments were re-assigned to internal fragments, based on exact mass measurement.
\end{abstract}

\section{Introduction}

The explanation of peptide fragmentation pathways is challenging and the development of powerful MS/MS techniques and new activation methods is crucial for proteomics. ${ }^{1} \mathrm{~A}$ better understanding of the involved mechanisms is important for the application of mass spectrometry in biochemistry and biology. Peptide dissociation mass spectra are difficult to predict, since fragmentation depends on numerous factors like charge state, amino acid composition and the fragmentation technique used. Ultraviolet photodissociation has proven to be a powerful tool for proteomics applications. ${ }^{2,3}$ Due to the high UV absorptivity of the peptide bond and aromatic side chains, ${ }^{4}$ a wide range of commercially available UV lasers has been used for laser induced dissociation (LID), like the $\mathrm{F}_{2}$ excimer at $157 \mathrm{~nm},{ }^{5-7}$ the ArF excimer at $193 \mathrm{~nm},{ }^{8,9}$ tunable optical parametric oscillator (OPO) systems, ${ }^{10,11}$ as well as the 4 th harmonic of Nd:YAG lasers at $266 \mathrm{~nm} .{ }^{12,13}$ Information about the amino acid sequence is obtained by analysing the resulting mass spectra.

Institut für Ionenphysik und Angewandte Physik, Leopold-Franzens-Universität Innsbruck, Technikerstraße 25, 6020 Innsbruck, Austria.

E-mail: martin.beyer@uibk.ac.at

$\dagger$ Electronic supplementary information (ESI) available: Fig. S1-S6 and Tables S1-S6 as described in the text. See DOI: 10.1039/c6cp08436b
UV and IR spectroscopy of protonated peptides ${ }^{14,15}$ are useful methods for gaining insight into electronic and geometric structure, in particular the protonation sites. The classical approach towards peptide fragmentation is collision induced dissociation (CID), where a large number of low energy collisions with a neutral gas like argon leads to fragmentation of the biomolecular ion. ${ }^{16,17}$ Infrared multiphoton dissociation (IRMPD) supplies the energy needed for fragmentation via a large number of infrared photons, typically supplied by a $\mathrm{CO}_{2}$ laser. ${ }^{18,19}$ The deposited energy per photon is low (about $0.1 \mathrm{eV}$ ), and the experiment has to be conducted in ultrahigh vacuum to avoid collisional deactivation. Upon ultraviolet photodissociation (UVPD), more energy can be deposited in the ion with a single photon, e.g. $6.2 \mathrm{eV}$ for an $\mathrm{ArF}$ laser at $193 \mathrm{~nm}$, which opens new fragmentation channels ${ }^{1}$ like side chain cleavages. This allows to distinguish between leucine and isoleucine..$^{20,21}$ Peptides composed of naturally occurring amino acids do not contain chromophores at wavelengths longer than $310 \mathrm{~nm} .^{3}$ Peptide bonds absorb photons in the $190 \mathrm{~nm}$ region $^{4}$ which is well suited for the application of ArF excimer lasers. The aromatic residues of tyrosine, phenylalanine and tryptophan serve as chromophores in the region of the quadrupled Nd:YAG wavelength at $266 \mathrm{~nm} .{ }^{11,12,18}$ Due to the technical difficulties associated with the use of UV optics and laser systems, new strategies to enhance photodissociation cross sections at specific wavelengths were devised in the last decade. ${ }^{22-24}$ Wilson and 
Brodbelt demonstrated the sequencing of peptides at $355 \mathrm{~nm}$ (tripled Nd:YAG laser) by attaching a UV absorptive chromophore. ${ }^{25}$ Mass spectra were dominated by a series of either $b$ or $y$ ions depending on the location of the chromophore ( $\mathrm{N}$ - or C-terminus). Another innovative derivatization method was developed by Julian and co-workers. ${ }^{26}$ They modified the free thiol form of cysteine in peptides by quinones, resulting in backbone cleavages at the modified site upon UVPD at $266 \mathrm{~nm}$.

Fourier transform ion cyclotron resonance mass spectrometry ${ }^{27}$ (FT-ICR MS) combined with electrospray ionization ${ }^{28}$ (ESI) is well suited for the investigation of biomolecules and their fragments. ${ }^{29}$ Fragment ions can be assigned with high confidence due to the very high mass resolving power of the technique.

The well-studied pentapeptide leucine enkephalin ${ }^{10,14,15,30-32}$ (LeuEnk, YGGFL) contains two aromatic chromophores at the tyrosine and phenylalanine residues, which enhance photofragmentation yields. A previous study by Dugourd and co-workers was performed at three different wavelengths, 220, 260 and $280 \mathrm{~nm},{ }^{10}$ which did not provide a complete picture on the wavelength dependence of individual fragment intensities. The Zwier group recently applied cryogenic UV and IR-UV double resonance photofragmentation to elucidate the three-dimensional structure of protonated YGGFL, ${ }^{15}$ covering a relatively narrow region around the tyrosine absorption from 35200 to $36000 \mathrm{~cm}^{-1}$ with high resolution. In the present study an optical parametric oscillator system is utilised to record a broadband photodissociation spectrum in the $225-300 \mathrm{~nm}$ region, which corresponds to 33333 to $44444 \mathrm{~cm}^{-1}$. All fragments are analysed as a function of wavelength to elucidate the potential influence of the electronically excited state on the product distribution. Utilizing the high resolution of the FT-ICR mass spectrometer, the assignment of all fragments was checked, and four LID fragments and one CID fragment had to be re-assigned.

\section{Experimental}

The experiments were performed on a BRUKER APEX Qe FT-ICR mass spectrometer equipped with a 9.4 $\mathrm{T}$ superconducting magnet, the combined ESI/MALDI ion source Dual Source II, and a Nanobay Console, shown schematically in Fig. S1 (ESI $\dagger$ ). Ions can be selected via a quadrupole mass filter prior to the hexapole collision cell, where they are thermalized in collisions with argon at room temperature before being transferred to the ICR cell via an electrostatic lens system. In the ICR cell, ions are stored and mass selected. Tunable laser light from an optical parametric oscillator (OPO) EKSPLA NT342B, operated at $20 \mathrm{~Hz}$ repetition rate with a pulse length of 4-6 ns, is introduced through a window at the rear end of the vacuum system into the ICR cell. Admittance of the laser beam to the ICR cell is controlled with an electromagnetic shutter placed in the beam path. Since the laser beam is not focused, the photon flux in the ICR cell can be estimated with an uncertainty of $20 \%$, which is determined by the shot-to-shot variations of the OPO in the UV region. The line width is specified as better than $8 \mathrm{~cm}^{-1}$ for wavelengths below $409 \mathrm{~nm}$. The wavelength is monitored by coupling scattered light of the OPO signal stage via an optical fiber into a grating spectrometer (Ocean Optics USB2000+).

Chemicals were purchased from Sigma Aldrich. Leucine enkephalin was dissolved in 1:1 water/methanol at a concentration of $100 \mu \mathrm{Mol} \mathrm{l}^{-1}$. $0.5 \%$ acetic acid was added to the mixture to improve ionization efficiency. The pressure in the hexapole collision cell was kept at around $4 \times 10^{-6}$ mbar. For LID ions where preselected with the quadrupole, followed by broadband chirp and resonant single frequency excitation of unwanted ions in the ICR cell. In case of collision induced dissociation (CID) only the most abundant isotope of LeuEnk was guided to the collision cell, where they were activated in energetic collisions with argon.

The pressure in the ICR cell was kept in the lower $10^{-9} \mathrm{mbar}$ region. A full cycle, ion accumulation, laser irradiation and detection, took 1-3 s depending on the applied storage parameters and laser irradiation times. 10 experiment cycles were averaged to provide reliable signal for fragment ions with small intensities.

In the absence of other loss channels, like ion molecule reactions, the photodissociation cross section $\sigma$ for single photon activation is given by eqn $(1)^{33}$

$$
I_{0}=\left(\sum_{i=0}^{n} I_{i}\right) \mathrm{e}^{-\sigma \Phi t}
$$

with the intensities of the precursor ion $I_{0}$ and the $n$ product ions $I_{i}$, the photon flux $\Phi$ and the irradiation time $t$. For a pulsed laser as used here, the modified eqn (2) is more convenient, with wavelength $\lambda$, the number of pulses $p$, the pulse energy $E$ per area $A$, the Planck constant $h$ and the speed of light $c$.

$$
I_{0}=\left(\sum_{i=0}^{n} I_{i}\right) \mathrm{e}^{-\frac{\sigma \lambda p E}{h c A}}
$$

Rearrangement leads to the total photodissociation cross section, eqn (3).

$$
\sigma=\ln \left(\frac{\sum_{i=0}^{n} I_{i}}{I_{0}}\right) \frac{h c A}{\lambda p E}
$$

The measured pulse energy $E$ was multiplied by $0.92^{2}$ to account for the loss of $8 \%$ per prism caused by passing through two $\mathrm{CaF}_{2}$ prisms used for beam steering. Cross sections are additive so the partial cross section of a single fragment is defined by eqn (4).

$$
\sigma_{i}=\sigma \frac{I_{i}}{\sum_{i=1}^{n} I_{i}}
$$

For the sequential absorption of two or three photons, as encountered in the present study, this analysis is slightly modified. Assuming that the absorption cross section does not change after photon absorption, the sequential absorption kinetics is treated with the same rate for each step. The term $\sigma \Phi$ in eqn (1) corresponds to the first order rate coefficient, and is numerically extracted from the data. 
At the position of the ICR cell, $3.3 \mathrm{~m}$ from the exit of the OPO system, the diameter of the laser beam is $11 \mathrm{~mm}$. At thermal cyclotron radii in the range of $100 \mu \mathrm{m}$, complete overlap of the laser beam with the ion cloud stored in the ICR cell is in principle possible. However, laser beam misalignment, space charge effects due to a too large number of ions in the cell or unintentional excitation of the selected ion can lead to incomplete overlap. Because of the large number of different fragments, high signal intensity had to be used in the present experiment. As a consequence, $15 \%$ of the ions were missed by the laser beam. This was accounted for in the analysis.

\section{Results and discussion}

In this section, mass spectra, absorption cross sections and photodissociation kinetics of ionized leucine enkephalin are shown. Protonated YGGFL was fragmented and sequenced by laser-induced dissociation and collision-induced dissociation. First and higher order fragments are identified by analysing the kinetics. Photodissociation cross sections are determined as a function of wavelength. All experiments were performed at room temperature.

\section{Laser induced dissociation}

Fragment assignment and sequencing. For peptide sequencing, a full fragment set of the same type has to be obtained, e.g. $\mathrm{b}_{1}-\mathrm{b}_{4}$ as illustrated in Scheme 1. To test whether this is feasible in our setup, protonated YGGFL was isolated and irradiated for $1 \mathrm{~s}$ at $44444 \mathrm{~cm}^{-1}$, or $225 \mathrm{~nm}$, the shortest wavelength accessible with our OPO system. Fig. 1 and Table S1 (ESI $\dagger$ ) show all sequence fragments observed. To rule out spontaneous post-isolation fragmentation, a control spectrum was recorded under identical conditions without laser irradiation, which showed no fragmentation.

Overall, a, b, and y fragments are most abundant, Fig. 2. This is in agreement with other experiments that showed protonation at the amide nitrogen to be favorable for decomposition. Charge directed dissociation then leads to the formation of a, b or $\mathrm{y}$ ions. ${ }^{1}$ Additional fragments are formed by photochemical pathways. ${ }^{24}$ All those fragments $\left(a_{1}-a_{4}, b_{1}-b_{4}, y_{1}-y_{4}\right)$ have been identified with a mass error smaller than $0.4 \mathrm{mDa} . \mathrm{c}_{1}, \mathrm{c}_{2}, \mathrm{c}_{3}, \mathrm{x}_{3}$ and $\mathrm{x}_{4}$ are also formed, with very low intensities for the latter two, and a relatively high mass error for the $c_{3}$ fragment of $4 \mathrm{mDa} . \mathrm{c}_{4}, \mathrm{x}_{1}, \mathrm{x}_{2}$ and $z$-fragments are not detected. There are strong peaks at the nominal mass of $\mathrm{x}_{1}, \mathrm{x}_{2}, \mathrm{z}_{1}$ and $\mathrm{z}_{2}$ fragments, which are, however, about $0.023 \mathrm{Da}$ off the exact sequence ion mass. A possible explanation would be the exchange of $\mathrm{NH}_{2}$ against $\mathrm{O}(\Delta m=0.0238 \mathrm{Da})$. Mass errors would be much smaller $(<3 \mathrm{mDa})$, but there are two reasons that such an exchange is not very probable. First, the $\mathrm{z}_{1}$ fragment does not contain any nitrogen, so the required exchange is impossible. Second, a substitution of $\mathrm{NH}_{2}$ for $\mathrm{x}$ and z-ions would require the exchange of a backbone nitrogen atom, since $x, y$, and $z$-fragments do not contain an amine group. Even though there is no known rule that suppresses the formation of these fragment types, and they have been measured frequently upon LID at $157 \mathrm{~nm}$ for other peptides, ${ }^{3}$ the difference between expected and measured mass is too big. Thus the fragments observed at the nominal mass of $\mathrm{x}_{1}, \mathrm{x}_{2}, \mathrm{z}_{1}$ and $\mathrm{z}_{2}$ must be reassigned. We succeeded to assign these to internal fragments, former $\mathrm{x}_{1}$ to $\mathrm{YGGF}$ int $3+\mathrm{H}$, former $\mathrm{z}_{1}$ to GGF int $4+\mathrm{H}$, former $\mathrm{x}_{2}$ to YGGFL int1, and former $\mathrm{z}_{2}$ to YGGF int1, Scheme 2 and Table S2 (ESI $\dagger$ ).

Some sequence fragments exhibit notably high signals, Fig. 2. Backbone fragmentation next to Tyr and Phe, the amino acids with aromatic side chains, is a well-known fragmentation pattern. ${ }^{34}$ Dugourd and co-workers photolyzed the aromatic side chain of phenylalanine and isolated the resulting $\mathrm{m} / \mathrm{z} 465$ ion for renewed LID at $220 \mathrm{~nm}$. Without the chromophore, no enhanced fragmentation at the $\mathrm{a}_{4}$ position was observed. ${ }^{10}$ Thus the high intensities of the $\mathrm{a}_{4}$ and $\mathrm{a}_{1}$ fragments are explained by direct bond cleavage due to the chromophores. Enhanced cleavage near the aromatic residues was also reported upon LID at $266 \mathrm{~nm} .^{13}$ While in LID, the $\mathrm{a}_{4}$ fragment is formed directly, in CID the $\mathrm{a}_{4}$ fragment is mainly formed by consecutive fragmentation of the $b_{4}$ ion. ${ }^{30}$ Since in LID, fragmentation occurs mainly around the two chromophores, it is not surprising that fragment ions near the $\mathrm{z}_{4}$ bond do not form frequently $\left(\mathrm{x}_{4}, \mathrm{~b}_{1}, \mathrm{y}_{4}, \mathrm{c}_{1}, \mathrm{a}_{2}, \mathrm{x}_{3}\right)$. The other sequence ions observed do not show any unexpected behaviour.

Several non-sequence fragments are observed, Scheme 2 and Table S3 (ESI $\dagger$ ). The ions at a mass to charge ratio of $m / z 91$ and

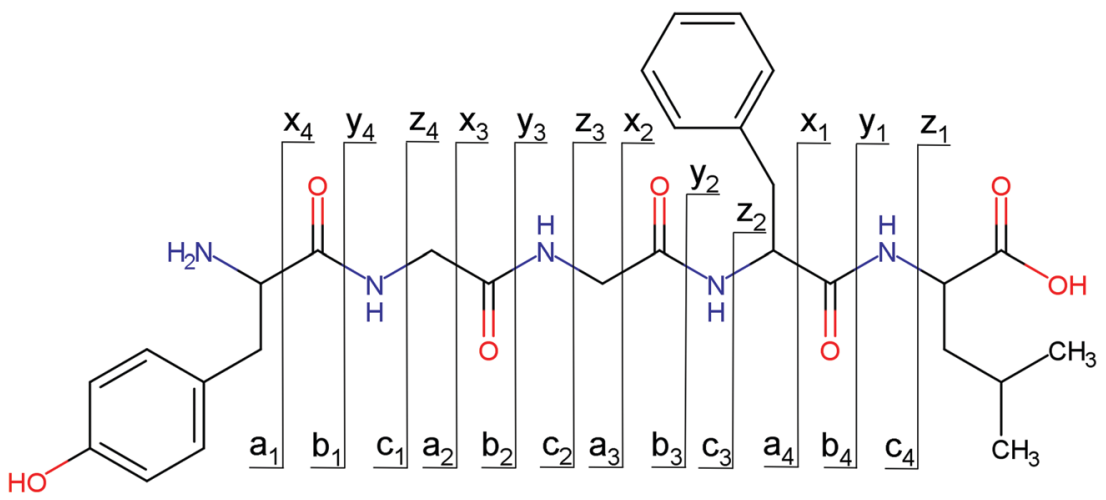

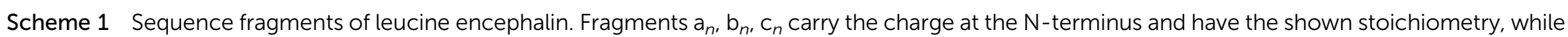
$x_{n}, y_{n}, z_{n}$ fragments carry the charge at the C-terminus of the peptide with two additional hydrogen atoms. 


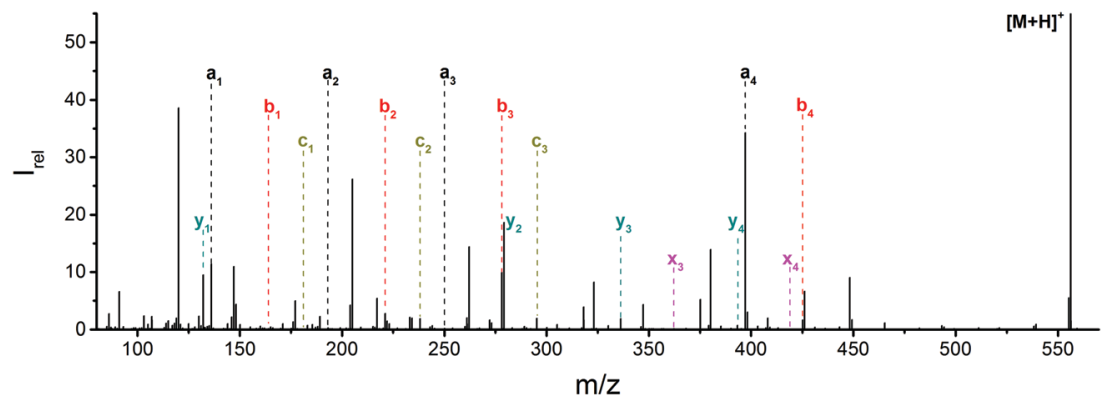

Fig. 1 Mass spectrum of leucine enkephalin upon LID at $44444 \mathrm{~cm}^{-1}$. Full sequence coverage for $a, b$, and y fragments has been achieved. Other intense fragments are discussed in the text.

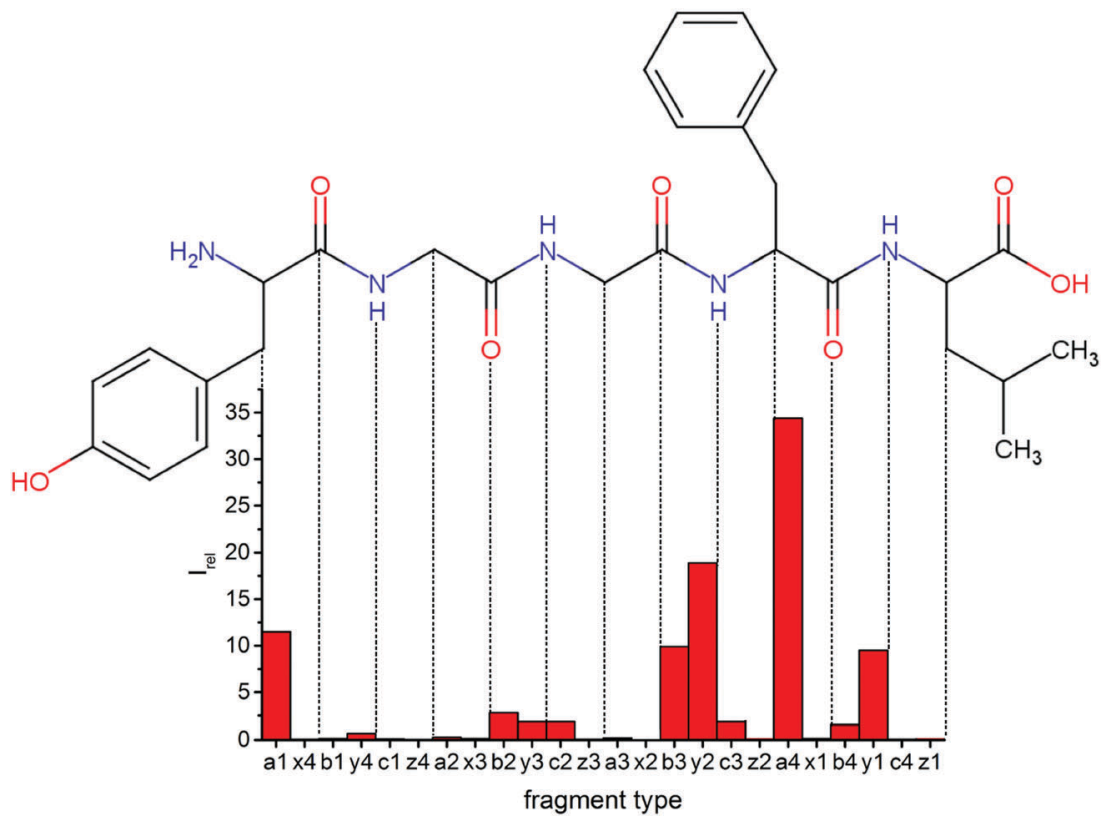

Fig. 2 Relative intensities of backbone fragments compared to precursor ion upon LID (protonated leuEnk, 15 laser pulses at $44444 \mathrm{~cm}^{-1}$ ). Most cleavage happens around the two chromophores, especially at the phenylalanine residue.

107 correspond to the phenylalanine and tyrosine aromatic side chain fragments, $\mathrm{F}$ asc and $\mathrm{Y}$ asc, respectively. Ions resulting from loss of the aromatic side chain at $m / z 465\left(\mathrm{MH}^{+}-\mathrm{F}\right.$ asc), 449 $\left(\mathrm{MH}^{+}-\mathrm{Y}\right.$ asc) and $448\left(\mathrm{MH}^{+}-\mathrm{Y}\right.$ asc $\left.-\mathrm{H}\right)$ are also observed. The peaks at $\mathrm{m} / \mathrm{z} 120$ and 136 represent the phenylalanine (F im) and tyrosine iminium ions ( $\mathrm{Y}$ im), respectively. In this case $\mathrm{Y}$ im and $\mathrm{a}_{1}$ are identical, since tyrosine is located at the $\mathrm{N}$-terminus of the peptide. Several internal fragments are detected: the ions at $\mathrm{m} / \mathrm{z}$ $147,162,177,205,219,234,262,290$ and 305 formed at the F chromophore are identified as GF int1, GF int2, GF int3, GGF int1, GGF int2, GGF int3, YGGF int1, YGGF int2, and YGGFL int1, respectively. In addition, sequence fragments with loss of water or ammonia are found, $y_{4}^{\circ}$ at $m / z 375$ and $a_{4}^{*}$ at $m / z 380$. The four remaining heavy fragments are: loss of a hydrogen atom $\mathrm{MH}^{+}-\mathrm{H}$, loss of formic acid $\mathrm{MH}^{+}-\mathrm{HCOOH}$, loss of $\mathrm{NH}_{3}$ and $\mathrm{HCOOH}$ $\mathrm{MH}^{+}-\left(\mathrm{NH}_{3}+\mathrm{HCOOH}\right)$ and loss of water $\mathrm{MH}^{+}-\mathrm{H}_{2} \mathrm{O}$. The latter three are intense for CID and will be discussed later. All nonsequence fragments are identified with a mass error smaller than $1 \mathrm{mDa}$. A complete list of fragment ions is provided as ESI. $\dagger$
Kinetics. To test how many photons are required to induce fragmentation and to determine which fragments arise from secondary fragmentation, protonated leucine enkephalin was irradiated at $225 \mathrm{~nm}$ with an increasing number of laser pulses, and LID mass spectra were recorded. The total photofragment intensity as a function of the number of laser pulses is shown in Fig. 3a. Already at 11 laser pulses $50 \%$ of the precursor ions are photodissociated, with about $15 \%$ of the precursor ions remaining at 40 pulses. Under ideal conditions, where each absorbed photon causes fragmentation and all ions are exposed to the same photon flux, the precursor intensity should decay exponentially. The semi-logarithmic plot in Fig. 3a shows obvious deviations from this ideal situation. There is a short induction period of about 5 laser pulses, during which the ions are heated so that an additional photon will be sufficient for fragmentation. After 30 laser pulses, the decay of the precursor ion intensity levels off, indicating that a fraction of $10-20 \%$ of the ions does not have trajectories that perfectly overlap with the laser beam. We assign this to space-charge effects due to 


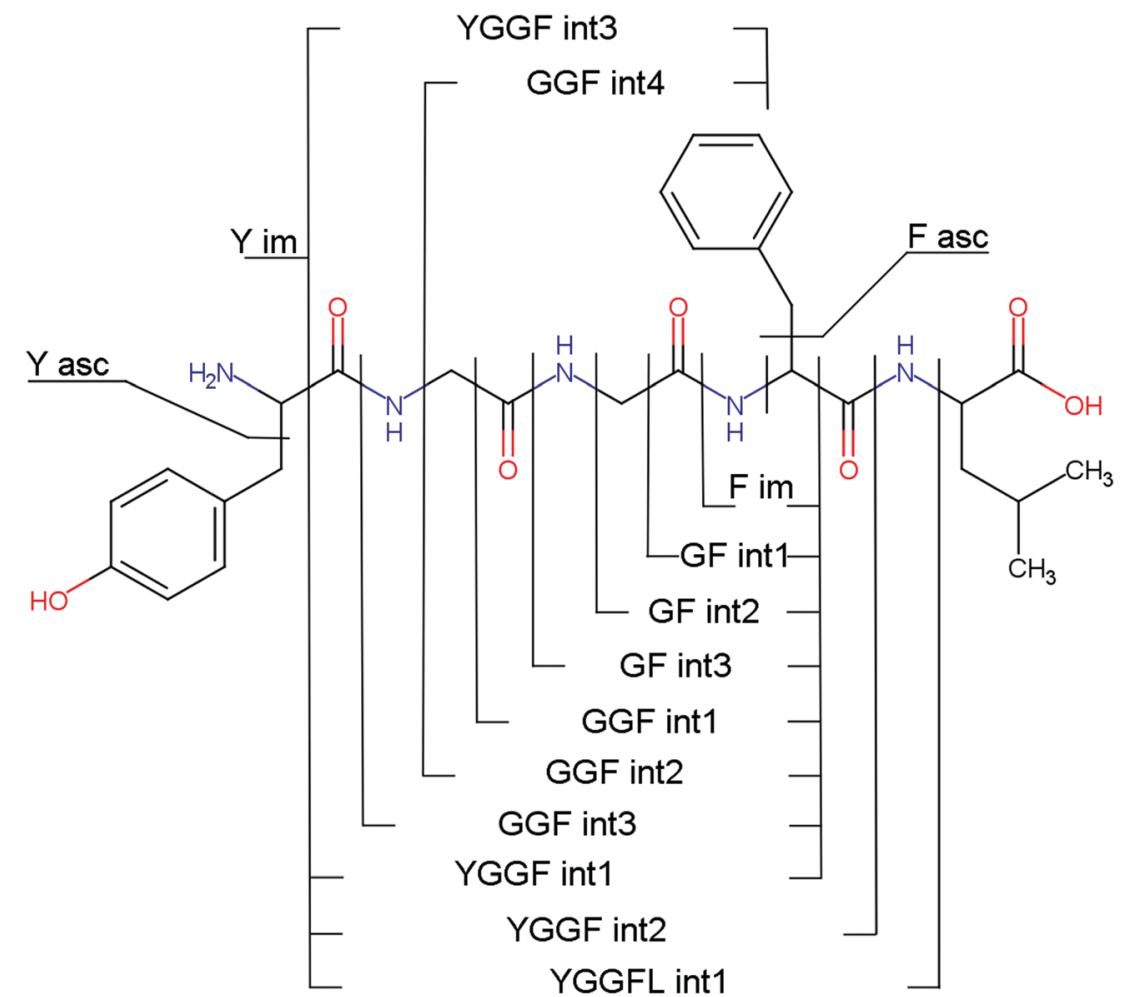

Scheme 2 Observed internal and side chain fragments. GF int1, GGF int1 and YGGF int1 could also form by breaking the $b_{4}-y_{4}$ or $c_{4}-z_{4}$ bonds. Since $a_{4}$ is the most abundant ion, the formation of these fragments is plausible. The stoichiometry of the aromatic side chain (asc) fragments is as shown, while internal fragments carry an additional proton.

the large number of precursor ions. We fitted the kinetics data assuming sequential absorption of one, two and three photons, see Fig. S2 (ESI $\dagger$ ). Most plausible are two and three photon processes.

Fig. 3b-d show the kinetics of a- and b-type fragments, aromatic side chain fragments, and the most abundant internal fragments, respectively. Most fragment ions already form upon the first laser pulse, which seems to be at odds with the induction period of 5 pulses described above. However, dissociation of the excited ion competes with infrared emission, so that the initially thermalized ions are difficult to dissociate. The internal energy increases with each absorbed photon, increasing the chance for dissociation.

Notable exceptions are the phenylalanine and tyrosine aromatic side chain ions $\mathrm{F}$ asc and $\mathrm{Y}$ asc, respectively, which are detected for the first time at 5 pulses with a steeply increasing intensity, Fig. 3c. This is consistent with their formation as fragments of the intense $\mathrm{a}_{4}$ and $\mathrm{a}_{1}$ ions, respectively. One might expect that also internal fragments requiring two bond cleavages are formed with a delay, but there is no evidence for such a behaviour in the data. However, one photon seems to be sufficient to photolyze the $\mathrm{C}-\mathrm{C}$ bond in the intact $\mathrm{MH}^{+}$precursor ion, since $\mathrm{MH}^{+}-\mathrm{F}$ asc and $\mathrm{MH}^{+}-\mathrm{Y}$ asc are observed already at the first laser pulse.

The phenylalanine iminium ion $\mathrm{F}$ im dominates the mass spectrum at 40 pulses due to higher order fragmentation, Fig. $3 \mathrm{~d}$. This is plausible concerning the preferred fragmentation at the aromatic side chain residues. ${ }^{10}$
Fig. $3 \mathrm{~b}$ shows the kinetics of a- and b-type ions relative to the precursor ion. $a_{2}, a_{3}$ and $b_{1}$ appear relatively late due to their low intensity, but the overall parallel behaviour to the more abundant sequence ions indicates that they are also formed as primary ions. The small $\mathrm{a}_{1}$ fragment, identical to $\mathrm{F} \mathrm{im}$, is abundant since it is located at the N-terminus of the peptide and contains the tyrosine chromophore. The intensities of $b_{2}$, $\mathrm{b}_{3}$ and $\mathrm{a}_{4}$ decline slowly after 10 laser pulses, indicating further fragmentation. This effect is most pronounced for $b_{3}$.

Photodissociation spectrum. Protonated leucine enkephalin was irradiated for 0.75 s, i.e. 15 laser pulses, from $225 \mathrm{~nm}$ to $250 \mathrm{~nm}$ in steps of $0.5 \mathrm{~nm}$ and from $250 \mathrm{~nm}$ to $300 \mathrm{~nm}$ in steps of $1 \mathrm{~nm}$. Due to the non-linear OPO process followed by frequency doubling to generate UV light, laser pulse energies vary in a range of $1.5-3.5 \mathrm{~mJ}$. Fig. 4 shows the total photodissociation spectrum of protonated YGGFL. Absolute cross sections were calculated assuming one, two, or three photon processes. Overall, the photodissociation spectrum of protonated YGGFL closely resembles the spectrum in aqueous solution, Fig. S3 (ESI $\dagger$ ), where the peptide is present as a zwitterion. Even the absolute absorption cross section is quite comparable, see ESI $\dagger$ for further details. The spectrum clearly exhibits two absorption maxima, which correspond to the $\mathrm{L}_{\mathrm{a}}$ and $\mathrm{L}_{\mathrm{b}} \pi-\pi^{*}$ transitions of tyrosine,$^{35}$ with a small contribution from the weaker $L_{b} \pi-\pi^{*}$ transition of phenylalanine.

The open source software Fityk ${ }^{36}$ was used to fit the data with Gaussian peaks. Regardless of the number of photons 

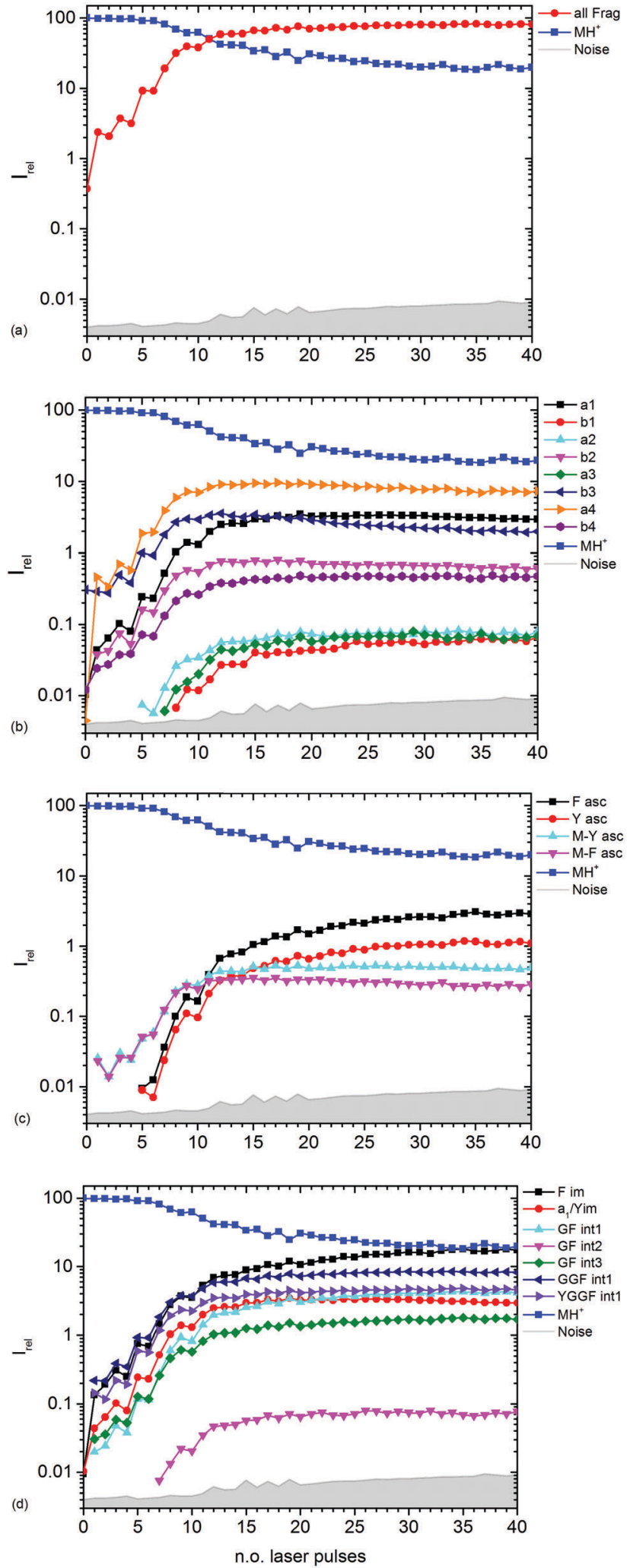

Fig. 3 Kinetics of different fragment types: (a) sum of all fragments, (b) a- and b-type fragments, (c) aromatic side chain fragments, (d) most abundant internal fragments.

assumed to be absorbed, three local photodissociation maxima are identified for the broad $\mathrm{L}_{\mathrm{b}}$ transition at $35452 \mathrm{~cm}^{-1}, 36696 \mathrm{~cm}^{-1}$

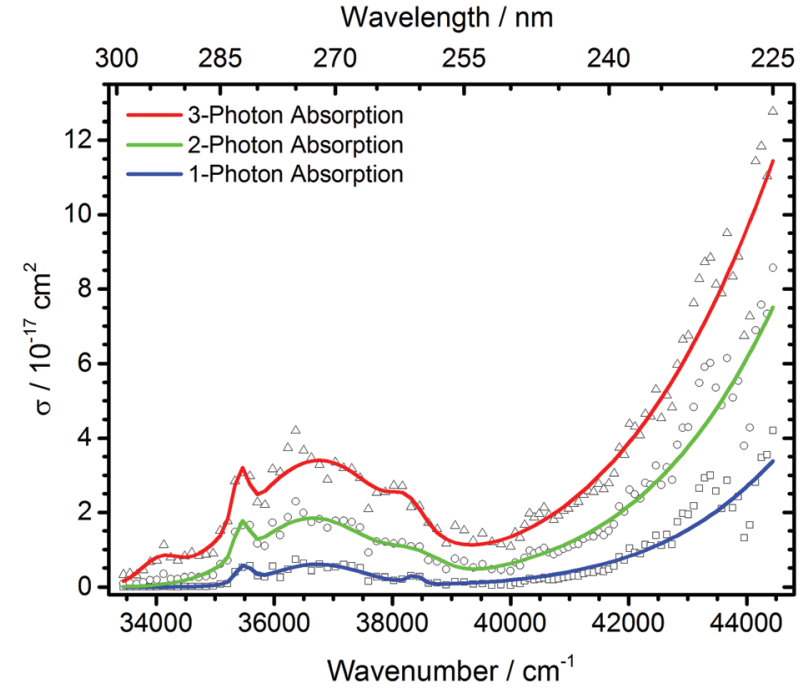

Fig. 4 Total photodissociation cross section of protonated leucine enkephalin calculated assuming sequential absorption of 1, 2 or 3 photons.

and $38355 \mathrm{~cm}^{-1}$. These may origin from different conformations of the protonated peptide. The maximum of the $\mathrm{L}_{\mathrm{a}}$ transition lies outside the OPO range, and was fixed to $52000 \mathrm{~cm}^{-1}$ to ensure a stable fit. It is very probable that also in this broad peak, different conformers contribute, but there is not sufficient information in the data to extract the relevant peak positions.

Other experiments showed strong fragmentation upon LID at $51813 \mathrm{~cm}^{-1}$ using an ArF excimer laser at $193 \mathrm{~nm}^{8,9}$ and $63694 \mathrm{~cm}^{-1}$ with an $\mathrm{F}_{2}$ excimer at $157 \mathrm{~nm}^{5-7}$ due to the high UV cross section of peptide bonds. ${ }^{4}$ The Zwier group performed UV and IR spectroscopy on cold protonated YGGFL. ${ }^{15}$ For the tyrosine chromophore, they found an absorption maximum around $35700 \mathrm{~cm}^{-1}$, which matches our experiments. The literature UV/VIS spectrum of tyrosine in solution ${ }^{37}$ shows a fast decline of absorption cross sections below $35600 \mathrm{~cm}^{-1}$, similar to our data. The Zwier group did not record a photofragmentation spectrum for the phenylalanine chromophore. UV laser-induced-fluorescence spectroscopy of bare phenylalanine reveals absorption bands in the $37500-37650 \mathrm{~cm}^{-1}$ region. ${ }^{38}$ This absorption should be present in our data. Due to the compared to tyrosine relatively small absorption cross section of phenylalanine, however, the F-chromophore is probably only a minor contribution. Oh et al. reported in a series of papers ${ }^{12,13,22}$ that for chromophorecontaining peptides, photodissociation is significantly enhanced at $266 \mathrm{~nm}$, i.e. $37600 \mathrm{~cm}^{-1}$, the wavelength of a frequency quadrupled Nd:YAG laser, consistent with our spectrum. No fragmentation is observed below $33500 \mathrm{~cm}^{-1}$.

The measured data points in part deviate significantly from the fit with Gaussian lines. This may be due to unresolved structure in our broad-band experiment with ions thermalized at room temperature. Close to the range limit of the OPO system, however, pulse-to-pulse variations of the laser power increase significantly, which may also contribute to the scattering of the data points around $44000 \mathrm{~cm}^{-1}$, and sequential multiphoton absorption increases this problem. 
Fig. 5 shows the branching ratio of characteristic fragments, averaged over three data points in narrow wavelength ranges at strong absorptions. The data for all fragments is available as ESI. $\dagger$ Some fragments are preferred at short wavelengths, like $\mathrm{H}$ atom loss (-H), YGGF int1, or YGGFL int1, but also sequence ions like $\mathrm{b}_{1}$. The sequence ions $\mathrm{a}_{4}, \mathrm{~b}_{4}$ and aromatic side chain loss $\mathrm{MH}^{+}$-Yasc dominate at long wavelengths, but also internal fragments like YGGF int2 may reach their highest branching ratio at longer wavelengths. Others, like $\mathrm{F}$ im or $\mathrm{y}_{2}$, have a nearly constant branching ratio throughout the photodissociation spectrum. This indicates that genuine photochemistry with bond cleavages or rearrangements in electronically excited states does play a role in LID of peptides at the investigated frequencies. The clearest case of direct photochemical bond cleavage is $\mathrm{MH}^{+}-\mathrm{H}$, which is only detected in the $\mathrm{L}_{\mathrm{a}}$ transition above $41000 \mathrm{~cm}^{-1}$. As pointed out by Dugourd and co-workers, ${ }^{10}$ it is most likely formed via internal conversion through a conical intersection between a ${ }^{1} \pi \pi^{*}$ state and a repulsive ${ }^{1} \pi \sigma^{*}$ state of the tyrosine aromatic side chain, as proposed by Sobolewski et $a l^{39}$ In close analogy to the mechanism of electron capture dissociation, ${ }^{40}$ the nascent $\mathrm{H}$ radical is mobilized and may induce bond cleavages along the peptide backbone, which would seamlessly explain the observation of the modified internal fragments YGGF int $3+\mathrm{H}$ and GGF int $4+\mathrm{H}$.

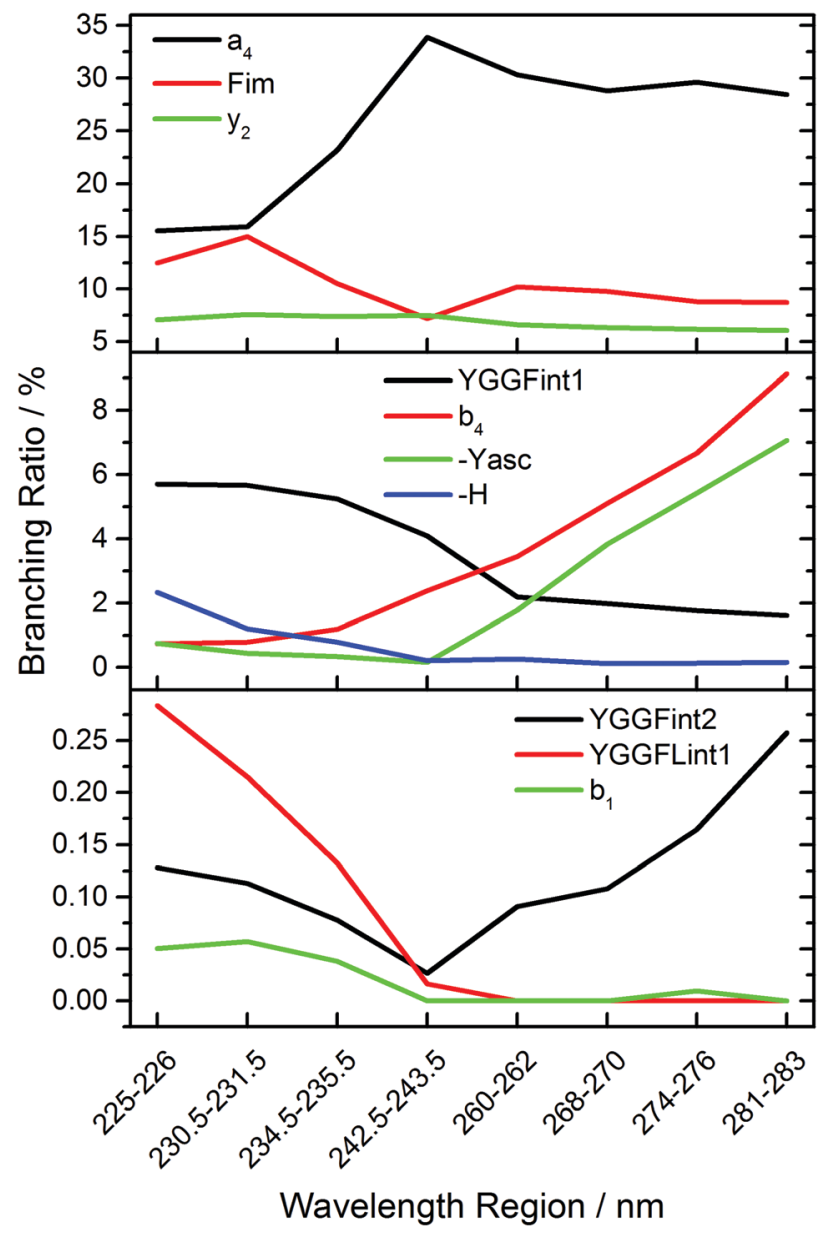

Fig. 5 Branching ratio of selected fragments as a function of frequency.
However, the fact that at least two, and possibly three photons must be sequentially absorbed before dissociation occurs clearly indicates that rapid internal conversion occurs, in agreement with the well-known photochemical properties of aromatic biomolecules. $^{39}$ This makes statistical fragmentation from a vibrationally excited molecule in its electronic ground state not merely a plausible scenario, but rather the preferred dissociation pathway. A clear indication for this behaviour is the branching ratio of the $b_{4}$ fragment, which is significantly enhanced at longer wavelengths, while the $\mathrm{a}_{4} / \mathrm{b}_{4}$ ratio, Fig. $\mathrm{S} 4$, increases with decreasing wavelengths. This is consistent with the higher photon energy causing additional fragmentation of $b_{4}$, particularly above $40000 \mathrm{~cm}^{-1}$.

\section{Collision induced dissociation}

For comparison with the standard peptide fragmentation techniques, protonated leucine enkephalin was isolated with the quadrupole mass filter and collided with argon in the collision cell at elevated collision energies. The collision energy can be qualitatively controlled via a voltage offset applied to the radio frequency (RF) signal of the hexapole. Usually CID of peptides leads to different fragmentation patterns than LID, e.g. chromophore targeted dissociation for $\mathrm{LID}^{10}$ or almost exclusive formation of $\mathrm{a}, \mathrm{b}$ and $\mathrm{y}$ fragments for CID. ${ }^{8}$

Fragment assignment and sequencing. Fig. 6 shows the collision induced dissociation mass spectrum of protonated leucine enkephalin. Full sequence coverage has been achieved for $\mathrm{a}$ and $\mathrm{y}$ ions. Even though $\mathrm{b}_{4}$ is formed abundantly, no $\mathrm{b}_{1}$ fragments are detected. Out of all $x$ - and z-type fragments only $\mathrm{x}_{4}$ has been observed. $\mathrm{c}_{1}$ has been clearly identified, while $\mathrm{c}_{2}$ and $c_{3}$ are observed with a relatively high deviation from the expected mass of $4 \mathrm{mDa}$, Table S5 (ESI $\dagger$ ). As for LID, $\mathrm{c}_{4}$ has not been observed. Overall, CID mass spectra exhibit less fragmentation than LID. The $b_{4}$ and $a_{4}$ fragments are most abundant as shown previously. ${ }^{30,41}$ In contrast to LID, there are fewer small fragments, but more non-sequence ions at higher masses, like $\mathrm{MH}^{+}$-SC, $\mathrm{MH}^{+}-\left(\mathrm{NH}_{3}+\mathrm{HCOOH}\right), \mathrm{MH}^{+}-\mathrm{HCOOH}$ or $\mathrm{MH}^{+}-\mathrm{H}_{2} \mathrm{O} . \mathrm{MH}^{+}-\mathrm{SC}$ denotes the ion $\mathrm{MH}^{+}-\left(\mathrm{NH}_{3}+\mathrm{HCOOH}+\mathrm{CO}\right)$. It has the same nominal mass as $\mathrm{MH}^{+}-\mathrm{F}$ asc but differs absolutely by $28 \mathrm{mDa}$, Table S6 (ESI $\dagger$ ). The aromatic side chain fragments $\mathrm{F}$ asc and $\mathrm{Y}$ asc are not observed, and neither are $\mathrm{MH}^{+}-\mathrm{F}$ asc, $\mathrm{MH}^{+}-\mathrm{Y}$ asc, $\mathrm{MH}^{+}-\mathrm{H}$, or GF int1 and GF int2. With current models, the whole fragmentation pattern is hard to explain as it appears that with formation of $a_{4}^{*}, \mathrm{a}_{4}, \mathrm{~b}_{4}, \mathrm{MH}^{+}-\mathrm{SC}, \mathrm{MH}^{+}-\left(\mathrm{NH}_{3}+\mathrm{HCOOH}\right)$, $\mathrm{MH}^{+}-\mathrm{HCOOH}, \mathrm{MH}^{+}-\mathrm{H}_{2} \mathrm{O}$, most fragmentation is localized around the C-terminus of the peptide. ${ }^{1}$

Fig. 7 shows relative intensities of backbone fragments. Compared to LID there are fewer sequence ions, since less energy is deposited per collision event than by photon absorption, and some fragmentation pathways which are possible upon LID will not open. $a_{4}$ and $b_{4}$ make up more than $90 \%$ of the fragment intensities.

Fragmentation at different collision energies. CID was performed at RF voltage offsets from 0 to $20 \mathrm{~V}$. The fragment intensities as a function of acceleration voltage are shown in 


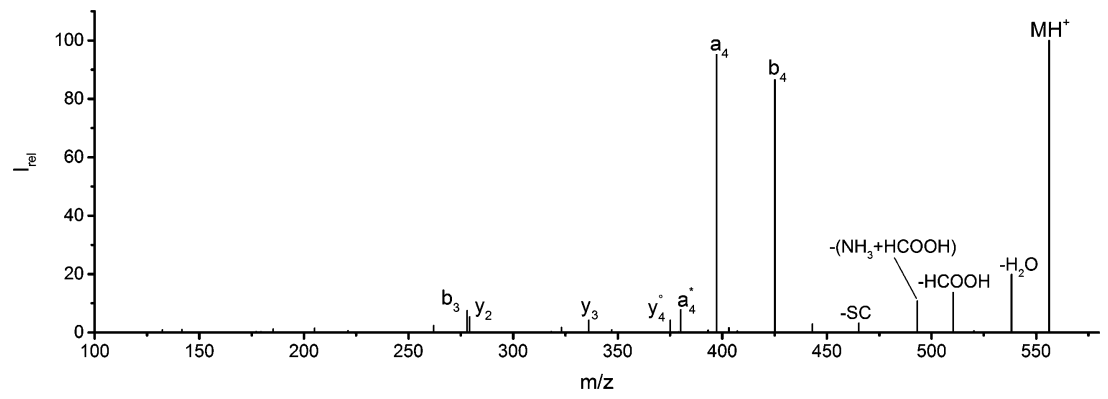

Fig. $6 \mathrm{CID}$ mass spectrum of protonated leucine enkephalin at $13.5 \mathrm{~V} \mathrm{CID} \mathrm{voltage.} \mathrm{Labeled} \mathrm{non-sequence} \mathrm{fragments} \mathrm{are:} \mathrm{loss} \mathrm{of} \mathrm{formic} \mathrm{acid} \mathrm{(-} \mathrm{HCOOH})$, loss of $\mathrm{NH}_{3}$ and $\mathrm{HCOOH}\left(-\left(\mathrm{NH}_{3}+\mathrm{HCOOH}\right)\right)$ and the loss of $\left(\mathrm{NH}_{3}+\mathrm{HCOOH}+\mathrm{CO}\right)(-\mathrm{SC})$.

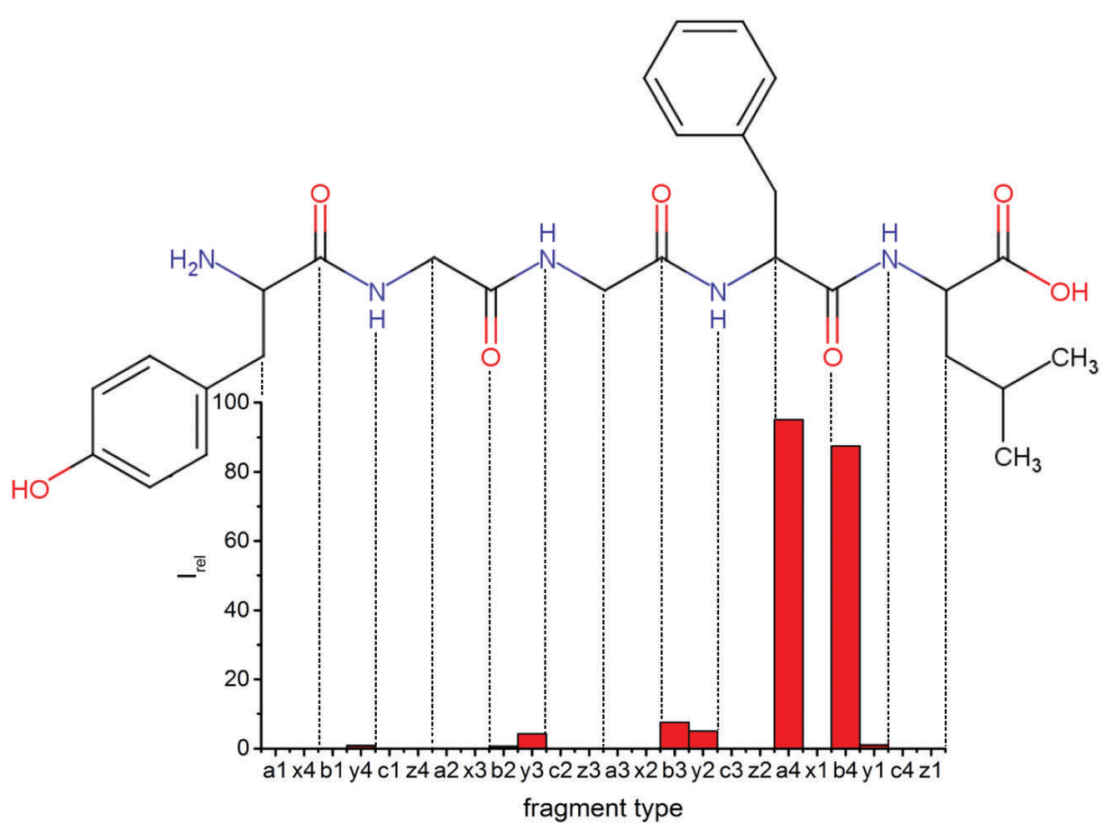

Fig. 7 Relative intensities of backbone fragments compared to the precursor ions for CID of protonated leuEnk, at $13.5 \mathrm{~V}$. For a qualitative comparison of ion signals with LID (Fig. 2) the experiment was performed at the CID voltage, where about the same amount of precursor ions remained.

Fig. 8 and Fig. S5 (ESI $\dagger$ ). Since only primary fragmentation takes place, each channel opens at a characteristic voltage. $\mathrm{a}_{4}$ and $\mathrm{b}_{4}$ are detected already below $2 \mathrm{~V} \cdot \mathrm{y}_{2}$ and $\mathrm{y}_{3}$ start forming at $4 \mathrm{~V}, \mathrm{~b}_{3}, y_{4}^{\circ}$ and $a_{4}^{*}$ at about $6 \mathrm{~V} . \mathrm{y}_{3}, \mathrm{~b}_{4}, \mathrm{M}^{+}-\left(\mathrm{NH}_{3}+\mathrm{HCOOH}\right)$, $\mathrm{M}^{+}-\mathrm{HCOOH}$ and $\mathrm{M}^{+}-\mathrm{H}_{2} \mathrm{O}$ decline at high acceleration voltages, Fig. 8a. The reasons are other preferred fragmentation pathways which require high energies, Fig. 8b. The loss of $\mathrm{H}_{2} \mathrm{O}$ is only observed between 6 and $20 \mathrm{~V}$. Low energy spectra are dominated by $b_{4}$ ions, high energy spectra by $a_{4}$. Aromatic side chain fragments are not detected. After $12.5 \mathrm{~V}$ more fragment ions than precursor ions are detected, Fig. S5a (ESI $\dagger$ ).

Fig. S5b (ESI $\dagger$ ) provides a closer look on a- and b-type ions and Fig. S5c (ESI $\dagger$ ) on internal fragments. $a_{1}, a_{2}$ and $a_{3}$ only form for CID voltages above $10 \mathrm{~V}, \mathrm{~b}_{3}$ and $\mathrm{b}_{2}$ form a little earlier, starting around $8 \mathrm{~V}$. There are fewer internal fragments in CID than in LID. These form at voltages above $7 \mathrm{~V}$. Since one additional bond has to be ruptured, a higher energy transfer into the precursor is required. The two iminium ions $\mathrm{F}$ im and $\mathrm{Y}$ im start at $10 \mathrm{~V}$ and behave almost identically.

\section{LID of mass selected fragments}

To identify important precursors for some of the secondary fragments, $\mathrm{MH}^{+}$was isolated in the quadrupole mass filter and collided with argon in the collision cell to generate $a_{4}$ and $b_{4}$ fragments, which were transferred into the ICR cell. The structure of these fragments has recently been studied with conformer-selective infrared-ultraviolet double-resonance photofragmentation by Wassermann $e t a l^{42}$ After mass selecting either $\mathrm{a}_{4}$ or $\mathrm{b}_{4}$, the ions were irradiated at $225 \mathrm{~nm}$ for $1 \mathrm{~s}$. The mass spectra shown in Fig. S6 (ESI $\dagger$ ) show that mostly small fragments are formed, which are not observed in CID mass spectra. Since there are two aromatic residues at the termini of $\mathrm{a}_{4}$ and $\mathrm{b}_{4}, \mathrm{~F}$ im and Y im are formed frequently, as only one bond has to be ruptured. $\mathrm{F}$ asc and $\mathrm{Y}$ asc are also observed, but with lower intensity. Also sequence ions are formed, $c_{3}$ predominantly from $a_{4}, b_{3}$ from $b_{4}$, and $b_{2}$ from both $a_{4}$ and $b_{4}$. The internal fragments GF int1 and GGF int3 with an additional loss of hydrogen are seen in both $a_{4}$ and $b_{4}$ spectra. Those fragments may also form directly. 

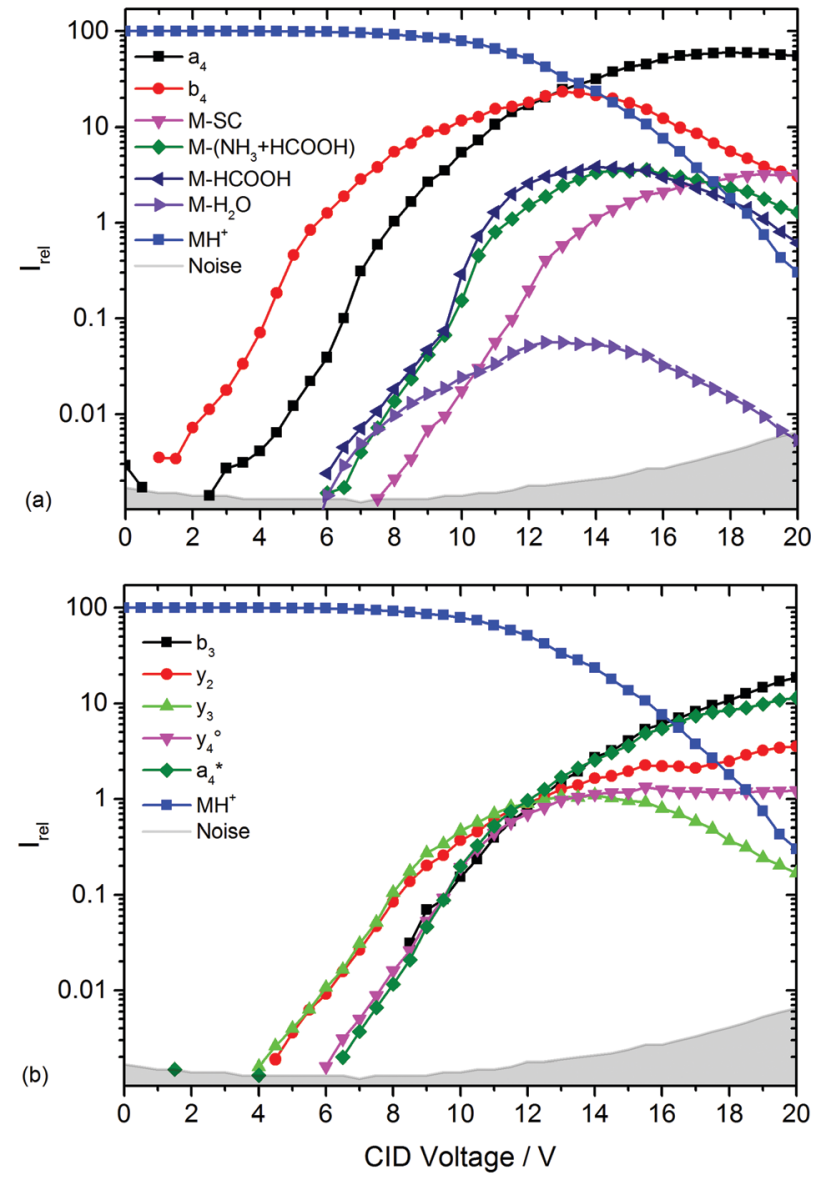

Fig. 8 Collision induced dissociation of protonated leucine enkephalin with argon for CID voltages from 0 to $20 \mathrm{~V}$. (a) Most abundant fragments $>397 \mathrm{Da}$, (b) most abundant fragments <397 Da.

\section{Conclusions}

Full sequence coverage of the protonated leucine enkephalin is obtained for $\mathrm{a}, \mathrm{b}$ and $\mathrm{y}$ fragments upon LID at $225 \mathrm{~nm}$. These ion types are most abundant and fragmentation is enhanced close to the phenylalanine and tyrosine aromatic side chain. In addition to the sequence ions, a variety of internal and side chain fragments are formed. Absorptions start around $33500 \mathrm{~cm}^{-1}$, strongly broadened due to the conformational flexibility of the peptide and the initial thermalization of the ions at room temperature. Two major maxima are identified, which correspond to the $\mathrm{L}_{\mathrm{a}}$ and $\mathrm{L}_{\mathrm{b}}$ transitions of $\pi-\pi^{*}$ type of the tyrosine chromophore, with minor contributions of the weaker $\mathrm{L}_{b}$ absorption of phenylalanine. The relative abundance of fragment ions depends in part strongly on the excitation wavelength. Internal conversion from the ${ }^{1} \pi \pi^{*}$ excited state via a repulsive ${ }^{1} \pi \sigma^{*}$ state to the ground state is followed by statistical unimolecular dissociation of the vibrationally excited molecular ion. To reach the threshold energy for dissociation in competition with radiative cooling, absorption of two to three UV photons is required. However, there is also clear evidence for excited state photochemistry, initiated by the loss of a hydrogen atom observed above $41000 \mathrm{~cm}^{-1}$. This hydrogen atom is released in the repulsive
${ }^{1} \pi \sigma^{*}$ state, and may induce further bond cleavages along the peptide backbone, similar to hydrogen atom induced bond cleavage in electron capture dissociation.

CID of the protonated leucine enkephalin by RF excitation in a hexapole collision cell leads to higher mass fragment ions than LID. The $a_{4}$ and $b_{4}$ fragments are most abundant and full sequence coverage is obtained for a and $\mathrm{y}$ ions. The loss of water, $\mathrm{HCOOH}$ or $\mathrm{HCOOH}$ together with $\mathrm{NH}_{3}$ are clearly visible. The difference between CID and LID lies in the different internal energy content of the molecular ion. In CID, multiple collisions add small amounts of energy, thus slowly approaching energy thresholds for dissociation. This favours dissociation pathways with low-lying tight transition states. In LID in the studied wavelength region, 4.3-5.5 eV are added upon absorption of a single photon. The overall larger energy content of UV excited molecular ions favours dissociation via high-lying lose transition states.

\section{Acknowledgements}

Start-up funds from the University of Innsbruck are gratefully acknowledged.

\section{References}

1 B. Paizs and S. Suhai, Mass Spectrom. Rev., 2005, 24, 508.

2 J. S. Brodbelt, Chem. Soc. Rev., 2014, 43, 2757.

3 J. P. Reilly, Mass Spectrom. Rev., 2009, 28, 425.

4 D. L. Peterson and W. T. Simpson, J. Am. Chem. Soc., 1957, 79, 2375.

5 T.-Y. Kim and J. P. Reilly, J. Am. Soc. Mass Spectrom., 2009, 20, 2334.

6 T.-Y. Kim, M. S. Thompson and J. P. Reilly, Rapid Commun. Mass Spectrom., 2005, 19, 1657.

7 A. Devakumar, M. S. Thompson and J. P. Reilly, Rapid Commun. Mass Spectrom., 2005, 19, 2313.

8 S. A. Robotham and J. S. Brodbelt, J. Am. Soc. Mass Spectrom., 2015, 26, 1570.

9 J. A. Madsen, T. S. Kaoud, K. N. Dalby and J. S. Brodbelt, Proteomics, 2011, 11, 1329.

10 T. Tabarin, R. Antoine, M. Broyer and P. Dugourd, Rapid Commun. Mass Spectrom., 2005, 19, 2883.

11 R. Antoine, M. Broyer, J. Chamot-Rooke, C. Dedonder, C. Desfrançois, P. Dugourd, G. Grégoire, C. Jouvet, D. Onidas, P. Poulain, T. Tabarin and G. van der Rest, Rapid Commun. Mass Spectrom., 2006, 20, 1648.

12 J. Y. Oh, J. H. Moon and M. S. Kim, Rapid Commun. Mass Spectrom., 2004, 18, 2706.

13 J. Y. Oh, J. H. Moon, Y. H. Lee, S.-W. Hyung, S.-W. Lee and M. S. Kim, Rapid Commun. Mass Spectrom., 2005, 19, 1283.

14 N. C. Polfer, J. Oomens, S. Suhai and B. Paizs, J. Am. Chem. Soc., 2007, 129, 5887.

15 N. L. Burke, J. G. Redwine, J. C. Dean, S. A. McLuckey and T. S. Zwier, Int. J. Mass Spectrom., 2015, 378, 196.

16 J. Laskin and J. H. Futrell, Mass Spectrom. Rev., 2003, 22, 158. 
17 J. M. Wells and S. A. McLuckey, Methods Enzymol., 2005, 402, 148.

18 W. Gabryelski and L. Li, Rapid Commun. Mass Spectrom., 2002, 16, 1805.

19 D. P. Little, J. P. Speir, M. W. Senko, P. B. O’Connor and F. W. McLafferty, Anal. Chem., 1994, 66, 2809.

20 L. Zhang and J. P. Reilly, J. Proteome Res., 2010, 9, 3025.

21 L. Zhang and J. P. Reilly, Anal. Chem., 2010, 82, 898.

22 J. Y. Oh, J. H. Moon and M. S. Kim, J. Mass Spectrom., 2005, 40, 899.

23 T. Tabarin, A. Kulesza, R. Antoine, R. Mitrić, M. Broyer, P. Dugourd and V. Bonacić-Koutecký, Phys. Rev. Lett., 2008, 101, 213001.

24 L. Vasicek and J. S. Brodbelt, Anal. Chem., 2010, 82, 9441.

25 J. J. Wilson and J. S. Brodbelt, Anal. Chem., 2007, 79, 7883.

26 J. K. Diedrich and R. R. Julian, Anal. Chem., 2010, 82, 4006.

27 A. G. Marshall, C. L. Hendrickson and G. S. Jackson, Mass Spectrom. Rev., 1998, 17, 1.

28 S. Banerjee and S. Mazumdar, Int. J. Anal. Chem., 2012, 2012, 282574.

29 Z. Guan, N. L. Kelleher, P. B. O’Connor, D. J. Aaserud, D. P. Little and F. W. McLafferty, Int. J. Mass Spectrom. Ion Processes, 1996, 157-158, 357.

30 R. W. Vachet, K. L. Ray and G. L. Glish, J. Am. Soc. Mass Spectrom., 1998, 9, 341.
31 X. Cai and C. Dass, Rapid Commun. Mass Spectrom., 2005, 19, 1. 32 F. Schinle, C. R. Jacob, A. B. Wolk, J.-F. Greisch, M. Vonderach, P. Weis, O. Hampe, M. A. Johnson and M. M. Kappes, J. Phys. Chem. A, 2014, 118, 8453.

33 B. Scharfschwerdt, C. van der Linde, O. P. Balaj, I. Herber, D. Schütze and M. K. Beyer, Low Temp. Phys., 2012, 38, 717.

34 L. Joly, R. Antoine, M. Broyer, P. Dugourd and J. Lemoine, J. Mass Spectrom., 2007, 42, 818.

35 L. H. Fornander, B. Feng, T. Beke-Somfai and B. Nordén, J. Phys. Chem. B, 2014, 118, 9247.

36 M. Wojdyr, J. Appl. Crystallogr., 2010, 43, 1126.

37 P. J. Linstrom and W. G. Mallard, NIST Chemistry WebBook, NIST Standard Reference Database Number 69, available at: http://webbook.nist.gov.

38 L. Snoek, E. Robertson, R. Kroemer and J. Simons, Chem. Phys. Lett., 2000, 321, 49.

39 A. L. Sobolewski, W. Domcke, C. Dedonder-Lardeux and C. Jouvet, Phys. Chem. Chem. Phys., 2002, 4, 1093.

40 F. W. McLafferty, D. M. Horn, K. Breuker, Y. Ge, M. A. Lewis, B. Cerda, R. A. Zubarev and B. K. Carpenter, J. Am. Soc. Mass Spectrom., 2001, 12, 245.

41 V. S. Rakov, O. V. Borisov and C. M. Whitehouse, J. Am. Soc. Mass Spectrom., 2004, 15, 1794.

42 T. N. Wassermann, O. V. Boyarkin, B. Paizs and T. R. Rizzo, J. Am. Soc. Mass Spectrom., 2012, 23, 1029. 